\title{
A new and greener method to manufacture copolymer-1
}

\author{
E. Ponnusamy \\ Sigma-Aldrich, USA
}

\begin{abstract}
Polyamino acid random copolymers have a wide variety of properties that mimic proteins, which make these random copolymers suitable for the treatment of certain diseases. For example, polyamino acid random copolymers comprising alanine, glutamic acid, lysine, and tyrosine (copolymer-1) have been used in the treatment of multiple sclerosis. Synthesis of this copolymer requires protecting groups for the side chains of the glutamic acid and lysine residues. The manufacture of copolymer-1 is typically achieved by protecting lysine with $\mathrm{N}^{\varepsilon}$ trifluoroacetyl (TFA) and glutamic acid with $\gamma$-benzyl protecting groups respectively. Deprotecting TFA and benzyl protecting groups require two separate steps. Removal of the benzyl-protecting group from glutamic acid requires using hazardous chemicals ( $\mathrm{HBr} /$ acetic acid) and highly flammable solvents and also generates a by-product, benzyl bromide a very strong lachrymator. Removal of the TFA group from lysine, the product needs to be further treated with flammable piperidine. These deprotection methods require long reaction times and handling, which in-turn reduces yield. Therefore, a need exists for an efficient, greener and less toxic process to manufacture copolymer1. A new greener method for the synthesis of Copolymer-1 has been developed. The method is greener, efficient, and uses less toxic reagents than literature methods. The present invention provides a method for the synthesis of copolymer-1 by polymerizing a mixture of $\mathrm{N}$-carboxyanhydrides of alanine, tyrosine, $\gamma$-ethyl-protected-glutamic acid, and $\mathrm{N}^{\varepsilon}$-TFA-protected-lysine in the presence of a polymerization initiator to form a protected polyamino acid random copolymer. Then adding a base to the protected polyamino acid random copolymer to cleave both the ethyl group from the glutamic acid and the TFA group from the lysine in a single deprotection step and also eliminated a large volume of hazardous chemical usage and waste generation. The new method
\end{abstract}


increased copolymer-1 yield, scalability, and quality and also reduced the cycle time.

Keywords: polyamino acid copolymers, protecting group, deprotection, dialysis, lyophilisation.

\section{Introduction}

Multiple sclerosis is a chronic inflammatory demyelinating disease of the central nervous system, in which infiltrating lymphocytes, predominantly T-cells and macrophages, cause damage of the myelin sheath. Copolymer-1 has been shown to alter positively the natural history of multiple sclerosis by both reducing the relapse rate and affecting disability [1-4]. It has been known to have suppressive and protective effects in experimental autoimmune encephalomyelitis, which can be induced in different species by various encephalitogenic antigens [5-9]. Copolymer-1, a synthetic polymer of amino acids, has a specific effect on the autoimmune process involved in experimental allergic encephalomyelitis and multiple sclerosis [10]. A major goal in the treatment of autoimmune disease has been the development of antigen-specific immunomodulating therapies [11].

Copolymer- 1 is a randomized mixture of synthetic polypeptides consisting of the four naturally occurring amino acids L-alanine, L-glutamic acid, L-lysine and L-tyrosine. The techniques used for the polymerization of the $\mathrm{N}$ carboxyanhydrides (NCA) to polymers are known to those skilled in the art and are given in detail in the review article by Goodman and Peggion [12], and the book by Kricheldorf [13] and also the recent publications by van Dijk-Wolthuis et al. [14]. The following synthesis specifies greener synthesis of poly (L-Ala, LGlu, L-Lys, L-Tyr) using N-carboxyanhydrides of L-alanine, $\gamma$-ethyl-L-glutamic acid, $\mathrm{N}^{\varepsilon}$-TFA-L-lysine and L-tyrosine.

\section{Experimental}

All materials used in this study were from Sigma-Aldrich. Varian Inova AS400 Nuclear Magnetic Resonance spectrometer (NMR) was used for NMR analysis. Dawn EOS by Wyatt Technologies was used for light scattering analysis and Cannon-Fenske viscometer, size 50 was used for viscosity measurements. Amino acid analysis was done using Pickering PCX5200 with Hitachi L7400 detector.

\subsection{Greener synthesis of Poly (L-Ala, L-Glu, L-Lys, L-Tyr) Acetate (Copolymer-1)}

\subsubsection{Polymerization}

Poly (L-Ala, $\gamma$-Et-L-Glu, $\mathrm{N}^{\varepsilon}$-TFA-L-Lys, L-Tyr) is prepared as follows: $3.334 \mathrm{~g}$ (0.029 mole) of L-alanine NCA, $1.942 \mathrm{~g}(0.010$ mole $)$ of $\gamma$-ethyl-L-glutamic acid NCA, $6.479 \mathrm{~g}$ (0.024 mole) of $\mathrm{N}^{\varepsilon}$-TFA-L-lysine NCA and $1.000 \mathrm{~g}(0.005$ mole $)$ of L-tyrosine NCA are dissolved in 0.225 liter of 1,4-dioxane to make a $0.3 \mathrm{M}$ solution. Add $\sim 1.25 \mathrm{~g}$ of charcoal and filter to get clear colorless solution. The filtered NCA solution is transferred to a 1 liter three neck RB flask equipped 
with mechanical mixing and a water bath at a temperature of $25-30^{\circ} \mathrm{C} .2 .7 \mathrm{ml}$ of $1 \mathrm{~N}$ sodium methoxide $(0.0027$ moles $)$ is placed in $25 \mathrm{ml}$ of 1,4 -dioxane. The sodium methoxide solution is added to the NCA solution in one portion with vigorous mixing. Polymerization proceeded at room temperature $\left(25-30^{\circ} \mathrm{C}\right)$ with evolution of carbon dioxide and the polymer solution is mixed for 2 hours and held at $25-30^{\circ} \mathrm{C}$ for $18-24$ hours.

\subsubsection{Precipitation of protected polymer}

Slowly pour the polymer solution in $1,250 \mathrm{ml}$ of DI-water with vigorous mixing. Protected polymer precipitates out and mix for 30 minutes. The product is filtered and washed the polymer with DI-water.

\subsubsection{Deprotection of ethyl and TFA groups}

Transfer the filtered polymer to a 500ml Erlenmeyer flask. Add $140 \mathrm{ml}$ of $1.5 \mathrm{M}$ ethanolic sodium hydroxide and mix for 0.5 hour. Polymer goes into solution within 5 minutes of adding the reagent. Dilute the polymer solution with $\sim 150 \mathrm{ml}$ of DI-water and mix for 5 minutes.

\subsubsection{Dialysis and lyophilization (freeze drying)}

Dialyze the deprotected polymer solution (18-24 hours) against running deionized water using $\sim 12 \mathrm{~K}$ molecular weight cut-off dialysis tubings. This process removes the oligomers, low molecular weight polymers and salts. The dialysis tubes are transferred to $3.5 \%$ acetic acid solution $(\sim 18 \mathrm{~L})$ and let stand for 7 hours and slowly mix the solution containing the dialysis tubings to make salt exchanges. Then dialyze the solution against running de-ionized water for 18-24 hours to remove the excess acetic acid, collect the solution, filter through a $0.2 \mu \mathrm{m}$ filter and lyophilize (freeze dry) to get the solid poly (L-Ala, L-Glu, LLys, L-Tyr) acetate (copolymer-1). Yield: 6.21g.

\section{Results and discussions}

${ }^{1} \mathrm{H}$ and ${ }^{13} \mathrm{C}$ NMRs showed the complete removal of the ethyl and TFA groups and also consistent with structure (Figures 1,2). Measured specific viscosity in

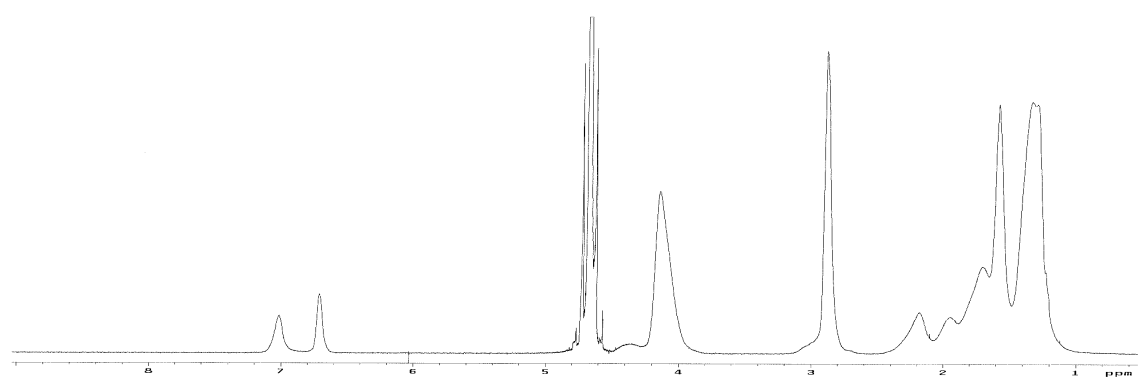

Figure 1: $\quad{ }^{1} \mathrm{H}$ NMR of copolymer-1. 
water, $1 \%$ solution at $25^{\circ} \mathrm{C}$ and calculated the viscosity molecular weight [15].

Also analyzed the polymer by light scattering (GPC MALLS) and determined molecular weight (Mw) (Figure 3). Molecular weight profile and polydispersity are similar to that of the old method. Measured specific rotation in water $(1 \%$ solution) at $25^{\circ} \mathrm{C}$ and the results are comparable. Amino acid ratios are determined by amino acid analysis and are within the expected range (Table 1).

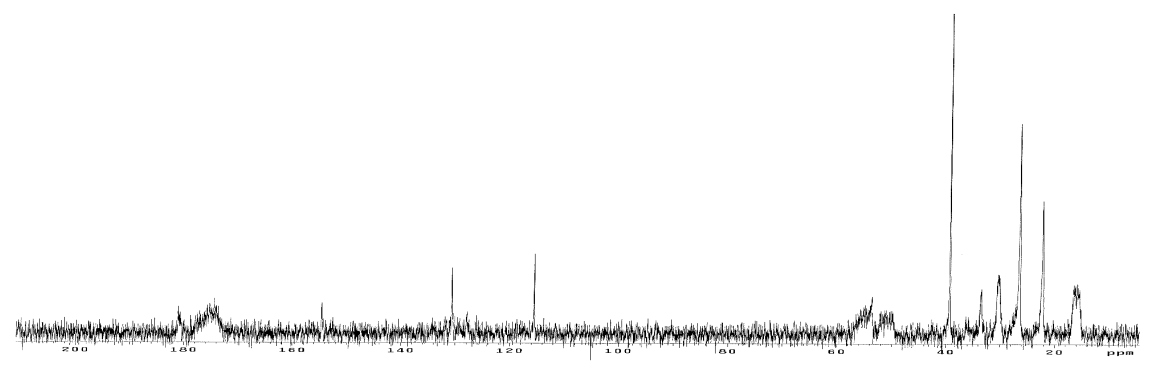

Figure 2: $\quad{ }^{13} \mathrm{C}$ NMR of copolymer-1.

Table 1: $\quad$ Molecular Weights, Polydispersity, Specific Rotation, Amino Acid Analysis and Yield for Copolymer-1.

\begin{tabular}{|c|c|c|c|c|c|}
\hline $\begin{array}{l}\text { Viscosity } \\
\text { Molecular } \\
\text { Weight }\end{array}$ & $\begin{array}{c}\text { GPC } \\
\text { MALLS } \\
\text { Molecular } \\
\text { Weight }\end{array}$ & $\begin{array}{l}\text { Polydispersity } \\
\text { (Mw/Mn) }\end{array}$ & $\begin{array}{l}\text { Specific } \\
\text { Rotation }\end{array}$ & $\begin{array}{c}\text { Amino } \\
\text { Acid } \\
\text { Analysis }\end{array}$ & $\begin{array}{c}\% \\
\text { Yield }\end{array}$ \\
\hline 18,000 & 21,300 & 1.2 & $-63.2^{\circ}$ & $\begin{array}{l}\text { Ala: } 5.70 \\
\text { Glu: } 1.90 \\
\text { Lys: } 4.80 \\
\text { Tyr: } 1.00\end{array}$ & 71.9 \\
\hline
\end{tabular}

Figure 3 shows the GPC MALLS profile for copolymer-1 and is consistent with the expected molecular weight and polydispersity.

The main purpose of the synthesis of copolymer-1 described above was to eliminate the usage of hazardous reagents ( $\mathrm{HBr} /$ acetic acid and piperidine) and make the process more greener and less labor intensive. Aqueous alkaline condition is used in the deprotection step of Poly (L-Ala, $\gamma$-Et-L-Glu, N${ }^{\varepsilon}$-TFA-LLys, L-Tyr) copolymer to remove the TFA group from lysine and the ethyl group from glutamic acid in a single reaction. No racemization occurs during the removal of these protecting groups, as is apparent from a comparison of the specific rotation of copolymer-1 prepared via benzyl protected glutamic acid $\left(-65^{\circ} \pm 5^{\circ}\right)$ with that of ethyl protected (Table 1$)$. 


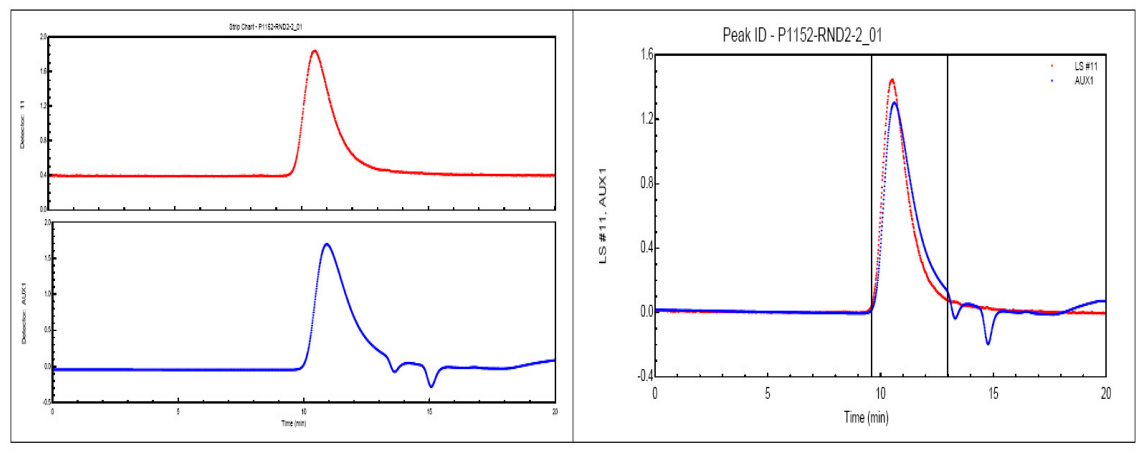

Figure 3: GPC MALS analysis instrument type: DAWN EOS; Cell type: K5; Laser wavelength: $690.0 \mathrm{~nm}$; Solvent: 20mM Sodium Phosphate $+130 \mathrm{mM}$ Sodium Chloride.

\section{Conclusions}

General procedure to make copolymer-1 was to polymerize Ncarboxyanhydrides of L-alanine, $\gamma$-benzyl-L-glutamic acid, $\mathrm{N}^{\varepsilon}$-TFA-L-lysine and L-tyrosine to make protected copolymer. Then remove benzyl group from glutamic acid with $\mathrm{HBr}$ /acetic acid and TFA group from lysine with piperidine in multiple steps and also a large volume of hazardous wastes have been generated. In the new process, eliminated the usage of hazardous $\mathrm{HBr}$ /acetic acid and piperidine reagents by replacing benzyl with ethyl protecting group for glutamic acid followed by a simple one step base deprotection. Also the new process eliminated large volume of hazardous waste generation. The new greener process decreased the cycle time to half, decreased the manufacturing cost, provided an unlimited scalability potential and also increased the yield without compromising quality.

\section{References}

[1] Johnson K. P., J. Neurol., 243, (Suppl. 1), S3-S7 (1996).

[2] Teitelbaum D., Milo R., Arnon R. and Sela M., Proc. Natl. Acad. Sci. USA, 89, 137-141 (1992).

[3] Comi G. and Moiola L., Baillieres Clin. Neurol. Oct. 6 (3), 495-509 (1997).

[4] Teitelbaum D., Arnon R. and Sela M., Cell Mol. Life Sci. 53, 24-28 (1997).

[5] Ziemssen T., Kumpfel T., Klinkert, W.E.F., Neuhaus, O. and Hohlfeld, R., Brain 125, 2381-2391 (2002).

[6] Teitelbaum D., Webb C., Meshorer A., Arnon R. and Sela M., Nature 240, 564-566 (1972).

[7] Web C., Teitelbaum D., Abramsky O., Arnon R. and Sela M., Isr. J. Med. Sci. 11, 1388 (1975). 
[8] Teitelbaum D., Fridkis-Hareli M., Arnon R. and Sela M., J. Neuroimmunol. 64, 209-217 (1996).

[9] Sela M., Int. Rev. Immunol. 18, 201-216 (1999).

[10] Sela M., Polym. Adv. Technol. 13, 859-862 (2002).

[11] Fridkis-Hareli M., Santambrogio L., Stern J.N.H., Fugger L., Brosnan C. and Strominger J.L., J. Clin. Invest. 109, 1635-1643 (2002).

[12] Goodman M. and Peggion E., Pure and Applied Chemistry, 53, 699-714 (1981).

[13] Kricheldorf H. R., “Alpha Amino Acids-N-Carboxyanhydrides and Related Heterocycles", Springer Verlag (1987).

[14] van Dijk-Wolthuis W. N. E., van de Water L., van de Wetering P., van Steenbergen M. J., Kettenes-van den Bosch J. J., Schuyl W. J. W. and Hennink W. E., Macromol. Chem. Phys. 198, 3893-3906 (1997).

[15] Yaron A. and Berger A., Biochim. Biophys. Acta 69, 397-399 (1963). 